\title{
Challenges in Laparoscopic Simple Nephrectomy of Non-functioning Kidneys Due to Urolithiasis
}

\author{
(1) Güner Yıldız', (1) Özcan Kılıç2, (1) Ali Furkan Batur2, (1) Murat Akand ${ }^{3}$ \\ 1 University of Health Sciences, Izmir Dr. Suat Seren Chest Diseases and Surgery Training and Research Hospital, Clinic of Urology, Izmir, Turkiye \\ 2Selçuk University Faculty of Medicine, Department of Urology, Konya, Turkiye \\ ${ }^{3} \mathrm{KU}$ Leuven, Department of Urology, Flanders, BE Leuven, Belgium
}

\section{What's known on the subject? and What does the study add?}

Laparoscopic simple nephrectomies due to atrophic kidneys may be challenging owing to the presence of fibrotic and inflammatory adhesions. Especially, nephrectomies for xanthogranulomatous pyelonephritis are associated with various difficulties. This study found that xanthogranulomatous pyelonephritis was the main pathology of all cases converted to open surgery. In addition, 19\% of the complications had Clavien-Dindo score $>1$, which is relatively less than reported in the literature.

\section{Abstract}

Objective: Simple nephrectomies may be challenging owing to the fibrotic and inflammatory processes that extend to the renal hilum and perirenal adipose tissue. This study aimed to investigate the complications of laparoscopic nephrectomies for atrophic kidneys.

Materials and Methods: Data of patients who underwent laparoscopic nephrectomy for atrophic kidneys were retrospectively evaluated. Preoperatively, all patients had undergone biochemical examinations, computed tomography and scintigraphic studies. The localisation of the stone, affected side, size of the atrophic kidney and presence of fistula and abscess were evaluated radiologically in the preoperative period. The pathology results of the patients were also assessed.

Results: A total of 53 patients were included. Conversion to open surgery was necessary in 3 (6\%) patients. Pathology reports of these patients revealed xanthogranulomatous pyelonephritis. Moreover, 10 (19\%) patients had Clavien-Dindo score $>1$. Postoperative fistula or abscess formation was not observed in any patient. None of patients had dialysis or sepsis in the postoperative period. Compared with the preoperative data, postoperative creatinine and blood urea nitrogen values were significantly increased.

Conclusion: Although laparoscopic nephrectomy is a gold standard treatment option, especially for atrophic kidney, nephrectomies for xanthogranulomatous pyelonephritis can become challenging even for the most experienced surgeons. Thus, surgeons should be always ready to transition to open surgery and be aware of the occurrences of complications in the perioperative and early postoperative periods.

Keywords: Laparoscopy, nephrectomy, urolithiasis, atrophic kidney, xanthogranulomatous pyelonephritis

\section{Introduction}

The incidence of upper urinary tract stones is increasing worldwide (1). Clearly, minimally invasive treatment options have increased in parallel with technological developments in the last 30 years. However, still, in Turkey, many patients have not received treatment or have delayed treatment due to stonerelated diseases. In patients with severe renal impairment due to stone-related diseases, nephrectomy may be required in some cases. Nephrectomies were performed for recurrent urinary tract infection, pain, severe hydronephrosis (pouch kidney), abscess formation and fistula formation (2).

The level of surgical difficulty increases because of the fibrotic and inflammatory processes extending to the renal hilum and perirenal adipose tissue. Therefore, complications may be encountered more often in simple nephrectomies than in kidney

Correspondence: Ali Furkan Batur MD, Selçuk University Faculty of Medicine, Department of Urology, Konya, Turkiye Phone: +90 3322412184 E-mail: alifurkanbatur@gmail.com ORCID-ID: orcid.org/0000-0001-7945-7326

Cite this article as: Yildiz G, Kiliç Ö, Batur AF, Akand M. Challenges in Laparoscopic Simple Nephrectomy of the Non-functioning Kidneys Due to Urolithiasis. J Urol Surg J Urol Surg 2021;8(1):54-58.

๑Copyright 2020 by the Association of Urological Surgery / Journal of Urological Surgery published by Galenos Publishing House. 
tumour nephrectomies because of the non-functioning kidneys. Perhaps, the most challenging procedure in this scenario is laparoscopic nephrectomy performed in patients with xanthogranulomatous pyelonephritis. In some cases, the chronic inflammatory process may have caused the development of squamous urothelial cancer, which had been overlooked with preoperative imaging techniques (3). The complication rates in these patients are higher than in patients who underwent nephrectomies for renal tumours. These complications include conversion to open surgery, bowel injuries (most importantly duodenum injury), solid-organ injuries (such as the liver and spleen), major vascular injuries (e.g. renal vessels, aorta, vena and cava), pneumothorax, port site infection and subcutaneous emphysema (3-5). If the procedure requires a laparoscopic approach, the surgeon must have a high level of experience.

Clayman described the first laparoscopic nephrectomy in 1991 (6). This procedure demonstrated shortened hospitalization time, short recovery time, less bleeding, postoperative pain as well as cosmetic advantages (7). Because of these advantages, the laparoscopic method has become the gold standard for nephrectomy for acute benign and malignant diseases $(2,8)$. However, simple nephrectomies performed by a laparoscopic approach can be very challenging and complicated than renal tumour nephrectomies, because of the aforementioned conditions. To our knowledge, only a few studies have examined the complications of simple nephrectomies and problems encountered in these surgeries (9). Thus, in this study, we aimed to investigate the difficulties and complications that we have encountered during laparoscopic simple nephrectomy for kidney atrophy due to urolithiasis.

\section{Materials and Methods}

Data of patients who underwent laparoscopic nephrectomy for atrophic kidneys between January 2013 and November 2018 were retrospectively evaluated. Informed consent was obtained for all patients. The local institutional review board approved the study (49109414-64.02).

All patients were over 18 years old, and their preoperative comorbidities and biochemical and radiological data were examined. Preoperatively, all patients underwent biochemical examinations, including renal function tests, complete urinalysis, urine culture examination, analysis of bleeding parameters, computed tomography (CT) and scintigraphic studies. Patients with positive urine culture were treated according to the antibiogram and then underwent surgery. In patients with clean urine culture, second-generation cephalosporin was administered as preoperative prophylaxis. Stone localization, affected side, size of the atrophic kidney and presence of fistula and abscess were evaluated radiologically in the preoperative period. Preoperative comorbidities were assessed according to American Society of Anesthesiologist (ASA) score (10), and perioperative and postoperative complications were evaluated according to the Clavien-Dindo Classification (11). The pathology results of the patients were also assessed.

All surgeries were performed through a transperitoneal laparoscopic approach. The standard laparoscopic nephrectomy procedure was performed with four trocars (one for the retraction of the liver) on the right side and three trocars on the left side. Renal pedicle vessels were clipped with hem-o-lock clips. Pathological specimens were usually removed by enlarging the incision through the trocar or using an Endo Catch specimen retrieval bag through the Pfannenstiel route.

\section{Statistical Analysis}

Statistical analyses were performed using the IBM Statistical Package for Social Sciences version 22 (IBM Corp., Armonk, NY, USA). Quantitative values are given as mean \pm standard deviation for parametric data, while quantitative values for nonparametric data are presented as median [minimum-maximum (min-max)]. Categorical variables are presented as numbers and percentages. Shapiro-Wilk and Q-Q plots were used to check the normality of the variables. Data were expressed as mean \pm standard deviation (range, min-max) or median (interquartile range) for continuous variables and described as counts (n) and percentages (\%) for categorical variables. Since the preoperative and postoperative haemoglobin values conformed to a normal distribution, the difference between the two values was compared with the significance test. Serum creatinine and blood urea nitrogen values were compared using the Wilcoxon test because they were not normally distributed in the Shapiro-Wilk test. A $p$-value of less than 0.05 was considered significant for all data.

\section{Results}

In total, 53 patients who underwent simple laparoscopic nephrectomy due to non-functioning kidneys were included. Scintigraphic evaluation data were available, and patients had $<10 \%$ of kidney functions. The mean age was 55 (39-73) years. The mean operation time was $153(75-215) \mathrm{min}$. The average body mass index was $28.3(23-35) \mathrm{kg} / \mathrm{m} 2$. Thirty (57\%) of the nephrectomies were performed on the left and $23(43 \%)$ were performed on the right side (Table 1). Conversion to open surgery was necessary in $3(6 \%)$ patients. Pathology reports of these patients revealed xanthogranulomatous pyelonephritis. However, preoperative CT results were not definitive for xanthogranulomatous pyelonephritis. In these cases, the renal pedicle could not be dissected because the renal hilum was very adherent. Therefore, open surgery was performed. Overall, four patients had Clavien-Dindo Illb complications. One patient had a 
colon injury, which was found intraoperatively and repaired by a general surgeon laparoscopically. One patient had minimal vena cava injury, which was endoscopically repaired and checked for haemostasis. One patient had a very minimal duodenal injury, which was repaired laparoscopically by the general surgeon. One patient developed ileus due to intestinal obstruction and underwent surgery to remove the bridges 72 hours later. As regards complications, 10 (19\%) patients had Clavien-Dindo score $>1$. Postoperative fistula or abscess development was not observed in any patient. None of the patients were on dialysis or had sepsis in the postoperative period. Blood transfusion was performed in three patients, and postoperative fever was also observed in three patients. No significant difference was found between the mean preoperative and early postoperative haemoglobin values [t $(52)=-1.284, p=0.205]$. Clavien-Dindo classification of the complications is summarised in Table 2 . In total, pathology reports of $48(91 \%)$ patients revealed chronic pyelonephritis. Xanthogranulomatous pyelonephritis was reported in 5 (9\%) patients (Table 1). Kidney function tests of the patients were checked at the third month after surgery;

\begin{tabular}{|c|c|}
\hline Characteristics & Patients \\
\hline \multicolumn{2}{|l|}{ Age (year) } \\
\hline Mean (min-max) & $55(39-73)$ \\
\hline \multicolumn{2}{|l|}{ Gender } \\
\hline Female & $33(62 \%)$ \\
\hline Male & $20(38 \%)$ \\
\hline \multicolumn{2}{|l|}{$\mathrm{BMI}\left(\mathrm{kg} / \mathrm{m}^{2}\right)$} \\
\hline Mean (min-max) & $28(23-35)$ \\
\hline \multicolumn{2}{|l|}{ Kidney } \\
\hline Right & $23(43 \%)$ \\
\hline Left & $30(57 \%)$ \\
\hline \multicolumn{2}{|l|}{ Renal Size, mm } \\
\hline Mean (min-max) & $107(69-133)$ \\
\hline \multicolumn{2}{|l|}{ ASA } \\
\hline 1 & $22(41 \%)$ \\
\hline II & $29(55 \%)$ \\
\hline III & $2(4 \%)$ \\
\hline IV & 0 \\
\hline \multicolumn{2}{|l|}{ Operation duration (min) } \\
\hline Mean (min-max) & $153(75-215)$ \\
\hline \multicolumn{2}{|l|}{ Pathology report } \\
\hline Chronic pyelonephritis & $48(91 \%)$ \\
\hline Xanthogranulomatous pyelonephritis & $5(9 \%)$ \\
\hline Conversion to open surgery & $3(6 \%)$ \\
\hline
\end{tabular}

the preoperative median creatinine level was 1.01 (0.78-1.23), and the postoperative level was $1.32(0.88-1.61)$, presenting a significant difference $(z=-6.301, p=0.000)$. Similarly, the median blood urea nitrogen levels in the preoperative and postoperative periods were 29 (14-41) and 33 (13-80), respectively, and the difference was significant $(z=-5.648, p=0.000)$.

\begin{tabular}{|l|l|}
$\begin{array}{l}\text { Table 2. Postoperative complications data according to the } \\
\text { Clavien-Dindo Classification }\end{array}$ \\
\hline Clavien-Dindo & N (\%) \\
\hline I & 0 \\
\hline II & $6(11 \%)$ \\
\hline IIla & 0 \\
\hline IIIb & $4(8 \%)$ \\
\hline IVa & 0 \\
\hline IVb & 0 \\
\hline V & 0 \\
\hline
\end{tabular}

\section{Discussion}

Urolithiasis is the most common benign disease that requires nephrectomy by impairing renal function. Recurrent infection, chronic pain and hypertension that cannot be corrected by antibiotic therapy necessitate nephrectomy in patients with urolithiasis $(12,13)$. However, laparoscopic nephrectomy can be much more challenging than radical nephrectomies. This is because recurrent infections cause an intense inflammatory reaction and the kidneys become adherent to the surrounding tissues. Besides, severe inflammatory reactions developed around the renal pedicle in these patients, which may make the dissection of the renal pedicle difficult and sometimes impossible via the laparoscopic route.

Laparoscopic surgery has become a gold standard treatment modality, especially for nephrectomy, in the last two decades. However, given the mentioned difficulties, serious complications can occur following laparoscopic simple nephrectomies. Recently, Zelhof et al. (2) analysed 1093 benign nephrectomies and reported the highest complication rate of $23.9 \%$ in patients with non-functioning kidneys caused by stones. In the same study, compared with radical nephrectomies performed only for T1 tumours, the overall complication rates (11.9\% vs 10\%), open surgery rates (5.9\% vs $3.3 \%$ ) and blood transfusion rates (4.8\% vs $2.8 \%$ ) were higher in benign nephrectomies (2). Again, in a recent study that included 149 patients, 19.3\% of the patients developed complications of Clavien-Dindo score $>1$, and the vascular injury rate was $3.3 \%$ (9). In our study, the overall complication rate was $19 \%$, that is, 10 patients had complications with Clavien-Dindo score $>1$. A vena cava injury with minimal area occurred in one patient during surgery, and it was repaired laparoscopically. 
Among other conditions, kidney size has been reported to increase the complication rates. Manohar et al. stated that the complication rate was high in patients $(n=84)$ with kidney size $>10 \mathrm{~cm}$ (14). In another study, the kidney size associated with increased risk of complications was $>12 \mathrm{~cm}$. In the present study, the mean kidney size was $107 \mathrm{~mm}$; however, when patients were evaluated in terms of complications, the kidney size was not a factor affecting the complication rate. In the literature, studies have indicated that the risk of complications significantly increases with a high ASA score, which is essential in predicting preoperative complications $(9,15)$. We cannot comment on this issue because only two of our patients had ASA 3 and none had ASA 4.

Conversion to open surgery is often observed in laparoscopic benign nephrectomies. Its rate is even higher in patients with xanthogranulomatous pyelonephritis. In a series of 62 patients, the rate of conversion to open surgery was $7.2 \%$ (3). In addition, this rate was as high as $28 \%$ in a series of 50 patients (16), but it was $19.2 \%$ in the study by Danilovic et al. (9). In their extensive research, Permpongkosol et al. (17) reported 5.9\% and 2.9\% for laparoscopic simple nephrectomy and radical nephrectomy in 2775 patients, respectively. In our study, the rate of conversion to open surgery was $6 \%$, and all pathology results of these cases revealed xanthogranulomatous pyelonephritis.

Radical nephrectomy is an independent risk factor for decreased renal function. Acute renal failure accounted for $0.4 \%$ of radical nephrectomy. However, few studies have evaluated renal function after nephrectomies for atrophic kidneys $(17,18)$. A study stated that $6.7 \%$ of patients who had undergone laparoscopic simple nephrectomy need dialysis in the first 6 months of follow-up (9). In our study, at 3 months postoperatively, both creatinine and blood urea nitrogen levels indicated a deterioration in renal functions.

\section{Study Limitations}

This study has certain limitations. First, we included a small number of patients. Second, our patients were not compared with those who underwent laparoscopic radical nephrectomies. Finally, the hospital where the surgeries were performed is not tertiary medical centre; this may have caused a tendency to perform surgery in patients with less comorbidity, albeit involuntarily. Although laparoscopic nephrectomy is a multidimensionally evaluated and established surgical method, very few studies have precisely assessed the complications of laparoscopic nephrectomies for non-functioning kidney urolithiasis; thus, the results of the present are valuable.

\section{Conclusion}

Although laparoscopic nephrectomy is a gold standard treatment option for nephrectomy, especially atrophic kidney, nephrectomies for xanthogranulomatous pyelonephritis can become challenging even for the most experienced surgeons. In our study, xanthogranulomatous pyelonephritis was found to be the main pathology of all cases converted to open surgery. However, preoperative CT results did not specifically present in any of these cases that the diagnosis could be xanthogranulomatous pyelonephritis. In the light of our study and the literature, laparoscopic simple nephrectomies performed primarily for xanthogranulomatous pyelonephritis can be challenging and complicated.

\section{Ethics}

Ethics Committee Approval: This retrospective study was approved by the institutional review board and was performed in accordance with the ethical standards.

Informed Consent: Informed consent was obtained for all patients.

Peer-review: Externally peer-reviewed.

\section{Authorship Contributions}

Surgical and Medical Practices: G.Y., Concept: G.Y., Design: G.Y., Data Collection or Processing: G.Y., A.F.B., Analysis or Interpretation: A.F.B., Literature Search: Ö.K., A.F.B., Writing: Ö.K., A.F.B., M.A.

Conflict of Interest: No conflict of interest was declared by the authors.

Financial Disclosure: The authors declare that they have no relevant financial.

\section{References}

1. Shoag J, Tasian GE, Goldfarb DS, Eisner BH. The new epidemiology of nephrolithiasis. advances in chronic kidney disease 2015;22:273-278.

2. Zelhof B, Mclntyre IG, Fowler SM, Napier-Hemy RD, Burke DM, Grey BR. Nephrectomy for benign disease in the UK: Results from the British Association of Urological Surgeons nephrectomy database. BJU Int 2016;117:138-144.

3. Angerri 0, López JM, Sánchez-Martin F, Millán-Rodriguez F, Rosales A Villavicencio H. Simple laparoscopic nephrectomy in stone disease: not always simple. J Endourol 2016;30:1095-1098.

4. Meraney AM, Samee AA EL, Gill IS. Vascular and bowel complications during retroperitoneal laparoscopic surgery. J Urol 2002;168:1941-1944.

5. Siqueira TM, Kuo RL, Gardner TA, Paterson RF, Stevens LH, Lingeman JE, Koch MO, Shalhav AL. Major complications in 213 laparoscopic nephrectomy cases: The Indianapolis experience. J Urol 2002;168:1361-1365.

6. Clayman R V., Kavoussi LR, Soper NJ, Dierks SM, Meretyk S, Darcy MD, Roemer FD, Pingleton ED, Thomson PG, Long SR. laparoscopic nephrectomy: Initial case report. J Urol 2017;197:S182-S186.

7. Rassweiler J, Fornara P, Weber M, Janetschek G, Fahlenkamp D, Henkel T, Beer M, Stackl W, Boeckmann W, Recker F, Lampel A, Fischer C, Humke U, Miller K. Laparoscopic nephrectomy: the experience of the laparoscopy working group of the German Urologic Association. J Urol 1998;160:18-21. 
8. Keeley FX, Tolley DA. A review of our first 100 cases of laparoscopic nephrectomy: defining risk factors for complications. Br J Urol 1998;82:615618.

9. Danilovic A, Ferreira TAC, Maia GV de A, Torricelli FCM, Mazzucchi E, Nahas WC, Srougi M. Predictors of surgical complications of nephrectomy for urolithiasis. Int Braz J Urol 2019;45:100-107.

10. Sankar A, Johnson SR, Beattie WS, Tait G, Wijeysundera DN. Reliability of the American Society of Anesthesiologists physical status scale in clinical practice. Br J Anaesth 2014;113:424-432.

11. Dindo D, Demartines N, Clavien PA. Classification of surgical complications: A new proposal with evaluation in a cohort of 6336 patients and results of a survey. Ann Surg 2004;240:205-213.

12. Mao $S$, Jiang $H$, Wu $Z$, Fang $Z$, Xia G, Ding Q. Urolithiasis: the most risk for nephrectomy in nonrenal tumor patients. J Endourol 2012;26:1356-1360.

13. Pearle MS, Goldfarb DS, Assimos DG, Curhan G, Denu-Ciocca CJ, Matlaga BR, Monga M, Penniston KL, Preminger GM, Turk TMT, James R White JR, American Urological Assocation. Medical management of kidney stones: AUA guideline. J Urol 2014;192:316-324.
14. Manohar T, Desai $M$, Desai M. Laparoscopic nephrectomy for benign and inflammatory conditions. J Endourol 2007;21:1323-1328.

15. Matin SF, Abreu S, Ramani A, Steinberg AP, Desai M, Strzempkowski B, Yang $Y$, Shen $Y$, Gill IS. Evaluation of age and comorbidity as risk factors after laparoscopic urological surgery. J Urol 2003;170:1115-1120.

16. Duarte RJ, Mitre Al, Chambô JL, Arap MA, Srougi M. Laparoscopic nephrectomy outside gerota fascia for management of inflammatory kidney. J Endourol 2008;22:681-686.

17. Permpongkosol $\mathrm{S}$, Link RE, Su L-M, Romero FR, Bagga HS, Pavlovich $\mathrm{CP}$, Jarrett TW, Kavoussi LR. Complications of 2,775 urological laparoscopic procedures: 1993 to 2005. J Urol 2007;177:580-585.

18. Huang WC, Levey AS, Serio AM, Snyder M, Vickers AJ, Raj GV, Scardino PT, Russo P. Chronic kidney disease after nephrectomy in patients with renal cortical tumours: a retrospective cohort study. Lancet Oncol 2006;7:735740 .

19. Stephenson AJ, Hakimi AA, Snyder ME, Russo P. Complications of radical and partial nephrectomy in a large contemporary cohort. J Urol 2004;171:130134. 